\title{
Food and You 2 - Wave 2
}

\author{
Area of research interest: Food and You 2 \\ Project status: Completed \\ Authors: Dr Beth Armstrong, Lucy King, Robin Clifford, Mark Jitlal \\ Conducted by: Ipsos MORI \\ Date published: 29 July 2021 \\ DOI: https://doi.org/10.46756/sci.fsa.dws750
}

\section{Introduction}

Food and You 2 is a biannual survey which measures self-reported consumer knowledge, attitudes and behaviours related to food safety and other food issues amongst adults in England, Wales, and Northern Ireland.

The survey is primarily carried out online using a methodology known as 'push-to-web'.

Fieldwork was conducted between 20 November 2020 and 21 January 2021. A total of 5,900 adults from 3,955 households across England, Wales and Northern Ireland completed the survey.

Topics covered in the Food and You 2: Wave 2 Key Findings report include:

- Trust in FSA and the food supply chain

- Concerns about food

- Food security

- Eating out and takeaways

- Food allergy, intolerance, and other hypersensitivities

- Food safety in the home

\section{Main findings}

Key findings from the Food and You 2: Wave 2 report:

- More than 9 in 10 (93\%) respondents reported that they had confidence that the food they buy is safe to eat. Over three quarters of respondents $(77 \%)$ reported that they had confidence in the food supply chain

- Three quarters (78\%) of respondents who had a least some knowledge about the FSA trust the FSA to make sure food is safe and what it says it is

- Most respondents (88\%) reported that they had no concerns about the food they eat. The most common prompted concerns, from a given list of food related issues, were the amount of sugar in food (60\%), food waste (60\%) and animal welfare $(57 \%)$

- Across England, Wales and Northern Ireland, $84 \%$ of respondents were classified as food secure (73\% high, $11 \%$ marginal) and $16 \%$ of respondents were classified as food insecure (8\% low, $7 \%$ very low)

- Three fifths $(60 \%)$ of respondents had eaten food which was ordered from a takeaway either ordered directly or via an online delivery company (e.g. Just Eat, Deliveroo, Uber Eats etc.) in the previous 4 weeks

- Most respondents (87\%) reported that they had heard of the Food Hygiene Rating Scheme. Of those, 51\% had checked the Food Hygiene Rating of a food business in the previous 12 
months

- Fewer than 1 in $10(9 \%)$ respondents reported that they had a food intolerance, $3 \%$ had a food allergy, $1 \%$ had coeliac disease and $1 \%$ had multiple food hypersensitivities

- Respondents who suffer a bad or unpleasant physical reaction to food were more likely to report confidence in the allergen information provided by restaurants $(82 \%)$, cafés, coffee or sandwich shops $(79 \%)$, and pubs or bars $(75 \%)$ compared to information provided by takeaways when ordering directly from a takeaway shop or restaurant $(63 \%)$ or when ordering through an online ordering and delivery company (e.g. JustEat, Deliveroo, UberEats) (50\%), food-sharing apps (e.g. Olio or Too Good To Go) (23\%), or Facebook Marketplace (21\%)

- More than 6 in $10(62 \%)$ respondents reported that they always check use-by dates before they cook or prepare food

\section{Research reports}

PDF

View Food and You 2 - Wave 2 - final report as PDF(Open in a new window) $(741.88 \mathrm{~KB})$ PDF

View Food and You 2 - Wave 2 - technical report as PDF(Open in a new window) (677.22 KB)

\section{Data tables}

The results tables for the Wave 2 report are available in our data catalogue. 